\title{
RETRACTED ARTICLE: Development of a neural network for glucose concentration prevision in patients affected by type 1 diabetes
}

\author{
Bartolomeo Cosenza
}

Received: 5 January 2012/Accepted: 2 May 2012/Published online: 24 May 2012

(C) Springer-Verlag 2012

This article "Development of a neural network for glucose concentration prevision in patients affected by type 1 diabetes (doi:10.1007/s00449-012-0750-0)" has been retracted by the Editor(s) because there are unresolved issues relating to authorship.

B. Cosenza $(\bowtie)$

Dipartimento di Ingegneria Chimica, Gestionale,

Informatica, Meccanica, Università di Palermo, Palermo, Italy

e-mail: bartolomeo.cosenza@unipa.it 